\title{
The Added Benefit of Hybrid SPECT/CT in the Diagnosis of Bone Metastasis in Patients with Breast Cancer
}

\author{
${ }^{1}$ Nasim Khan, ${ }^{2}$ SM Moinul Islam, ${ }^{2}$ Abrar Al Sakib, ${ }^{1}$ Saiyeeda Mahmood, ${ }^{1}$ GaziAbul Hossian, ${ }^{1}$ Ratan \\ Kumar Chakraborty, ${ }^{1}$ Nazmun Nahar, 1ShakilaZaman Rima, ${ }^{1}$ Md. Fakhar Uddin \\ ${ }^{1}$ Institute of Nuclear Medicine and Allied Sciences (INMAS), \\ Mymensingh Medical College Hospital Campus, Mymensingh, Bangladesh. \\ ${ }^{2}$ Department of Medicine, Mymensingh Medical College Hospital, Mymensingh.
}

For Correspondence: Nasim khan, Associate Professor/PMO, Institute of Nuclear Medicine and Allied Sciences (INMAS), Mymensingh Medical College Hospital Campus, Mymensingh

Bangladesh. E-mail: mnasim52@ hotmail.com

\begin{abstract}
The purpose of this study was to investigate the additional benefit of single photon emission computed tomography/computed tomography (SPECT/CT) over whole-body planar bone scintigraphy (planar BS) and SPECT for the detection of bone metastases in breast cancer patients. Materials \& methods: Of 112 consecutively examined patients with histologically confirmed breast carcinoma who underwent bone scintigraphy, 39 required further evaluation by SPECT/CT $(n=23)$ and SPECT alone $(n=16)$ because a definite diagnosis could not be established using whole body planar BS alone. The 23 SPECT/CT studies were retrospectively evaluated by two nuclear medicine physicians for planar BS and SPECT images, and one nuclear medicine physician and one radiologist for SPECT/CT on consensus. Each focus of abnormal tracer uptake was recorded for differentiating malignant from benign bone lesions. Clinical and imaging follow-up were used as a reference standard. Results: A total of 72 lesions were evaluated in 23 patients examined by three types of imaging modalities. In 57 of the 72 evaluated lesions, the results of planar BS, SPECT and SPECT/CT were concordant; 52 of the 57 lesions were interpreted as malignant and 5 lesions as benign by all modalities. In 15 of the 72 lesions, consensus reading of fused SPECT/CT images changed the image interpretation of 15 planar BS scans and 9 of the 15 SPECT scans: 7 lesions previously interpreted as benign (on both planar BS and SPECT) were re-classified as malignant and 8 lesions ( 8 by planar BS and $2 / 8$ by SPECT) previously interpreted as malignant were reevaluated as benign. The highest diagnostic gain was in the thoracolumbar spine, thoracic cage \& pelvis. The overall accuracy of SPECT/CT was significantly higher on a lesion-based analysis than planar BS and SPECT $(100 \%$ vs $79 \%, p<0.0001$ and $100 \%$ vs $83 \%, p=0.003)$. Compared to planar BS and SPECT, the results of SPECT/CT changed diagnosis or treatment in 5/23 patients $(21.7 \%)$.
\end{abstract}


Conclusion: The hybrid imaging system with SPECT/CT is a feasible technique yielding coregistered dual-modality images. The addition of SPECT/CT improves the diagnostic accuracy for the correct interpretation of bony lesions in breast cancer patients undergoing bone scanning for metastases. SPECT/CT should be routinely used in the work up of postoperative breast carcinoma patients with simultaneous CT evaluation to identify metastatic lesions not detected on bone scintigraphy.

Keywords: SPECT/CT, ${ }^{99 \mathrm{~m}}$ Tc-MDP, bone metastasis, breast cancer.

\section{INTRODUCTION}

Breast cancer is the commonest cancer in females worldwide. The skeleton is the most common site of distant metastases in breast cancer. Bone is the first site of metastasis in $26 \%-50 \%$ of patients with metastatic breast cancer and it may develop during the course of the disease in $30 \%$ $85 \%$ of these patients (1). There are various anatomical and functional imaging modalities for detecting and characterizing malignant bone involvement. Among them, planar bone scintigraphy has long been used as a valuable method for sensitively detecting or characterizing focal bone pathology; more recently, single- photon emission computed tomography (SPECT) has been used in this capacity (2). Although functional bone imaging is a highly sensitive method, it lacks specificity (3). Therefore, radiography, CT, or MRI is frequently performed after bone scintigraphy to further characterize lesions evident on bone scans. The recently introduced novel hybrid SPECT/CT system offers a direct correlation of focal bone pathology with anatomic structures and therefore minimizes the number of equivocal findings. Screening for bone metastases and evaluation of the treatment response are the most frequent indications for bone scintigraphy using ${ }^{99 \mathrm{~m}} \mathrm{Tc}$-methylene diphosphonate $\left({ }^{99 \mathrm{~m}} \mathrm{Tc}-\mathrm{MDP}\right)$. Although the majority of bone metastases appear as hot spots, some appear as cold lesions. Benign lesions, such as hemangioma, may also appear as cold, making the differential diagnosis problematic. The differentiation of benign and malignant lesions can usually be achieved with CT coregistration and is a major advantage of SPECT/CT (4). The initial results for diagnosis of skeletal metastasis with this combined functional and morphologic system have been promising (5). The prevalence and importance of accurate tumor staging makes carcinoma of the breast an interesting candidate for study. Furthermore, despite the increasing use of integrated SPECT/CT in the management of cancer patients, to our knowledge, the clinical utility of combined assessment of MDP avidity and morphologic changes in breast cancer metastases to bone has not been fully elucidated. Thus, the 
aim of the present study was to retrospectively investigate the additional benefit of MDPSPECT/CT over SPECT and planar imaging in breast cancer patients.

\section{MATERIALS AND METHODS}

\section{Patients}

One hundred and twelve post-operative patients with histologically confirmed breast carcinoma and available information on primary treatment underwent whole body bone scintigraphy in the context of suspected metastasis at the Institute of Nuclear Medicine \& Allied Sciences (INMAS), Mymensingh from December 2011 to August 2012. Approval from this institutional review board and written informed consent from the patients were obtained for this study. In 73 of the patients, planar BS showed no abnormal foci of radiotracer accumulation. In the remaining 39 patients, further work up by SPECT or SPECT/CT was necessary because of unclear findings of whole body planar BS. In 23 of these patients, a SPECT/CT scan was performed using a hybrid camera combining a dual-head gamma camera with dual-slice spiral CT scanner installed within the same gantry (Symbia T2; Siemens). In the other 16 patients, SPECT alone was performed, mainly because of the temporary technical defect of the SPECT/CT system. The 23 SPECT/CT studies were retrospectively evaluated by readers who were unaware of clinical pretest probability and the findings on the planar scintigraphy. The age range of patients was $44-76$ years (mean: 65 years). The bone lesions were considered metastatic if an increase in tumor size was confirmed with follow up imaging studies, including bone scintigraphy, CT and MRI, with or without exacerbation of clinical symptoms. According to these criteria, 59 lesions were considered metastatic bone lesions, and 13 were considered benign lesions (eg, spondylarthrosis, osteophyte, fracture) in these 23 patients.

\section{Image acquisition}

Planar bone scintigraphy was the first to be performed. Planar images of the entire skeleton were acquired 2-3 h after intravenous injection of ${ }^{99 \mathrm{~m}}$ Tc-MDP (mean dose, $735 \mathrm{MBq}$; range, 589-842 MBq) using a dual head gamma camera SPECT/CT system (Symbia T2; Siemens).After completion of planar scintigraphy, all 23 patients underwent SPECT with the gamma-camera used for scintigraphy. The detectors were positioned on the focal lesions localized with planar scintigraphy. The SPECT acquisition was performed in step-and-shoot mode over $360^{\circ}\left(180^{\circ}\right.$ per head) with the patient supine. A 128 x 128 matrix was used, with a 5.6 angular step, an acquisition time of $12 \mathrm{~s}$ per frame, and a zoom factor ranging from 1 to 1.2 according to the individual patient. SPECT data were reconstructed with an ordered subset expectation maximization (OSEM) algorithm using e.soft reconstruction software (Siemens). Immediately 
after SPECT acquisition, CT scan was performed keeping the patients at the same position. Unenhanced CT was acquired in helical mode with a table speed of $10.0 \mathrm{~mm} /$ rotation and a rotation time of 0.8 seconds. A voltage of $130 \mathrm{KV}$ was used, and current was automatically adjusted in the range of 100-700 mA, depending on the setting of a noise index ranging from 24 to 33 and 5-mm slices were obtained. After reconstruction, SPECT images were corrected for attenuation and scatter. Both SPECT and CT axial 5-mm slices were generated using an e.soft Syngo MI ApplicationsVA60C (Siemens) for evaluation. The SPECT/CT images were also analyzed using 2-dimensional orthogonal reslicing in axial, sagittal, and coronal orientations. A maximum- intensity projection 3-dimensional display was generated to improve contrast enhancement of the images.

\section{Image interpretation}

Planar scintigraphy, SPECT and SPECT/CT images were evaluated blindly and separately: first the planar BS images, then SPECT images by two nuclear medicine physicians in consensus, and last the fused SPECT/CT images by a nuclear medicine physician and a radiologist in consensus.

Each site of abnormally increased uptake of ${ }^{99 \mathrm{~m}}$ Tc-MDP was recorded and categorized as normal (no increased uptake), benign, malignant, or equivocal. Scintigraphic lesions were categorized as benign when they appeared as areas of low intensity increased tracer uptake or when they were located around joints. Vertebral lesions were considered malignant when they involved the posterior aspect of the vertebral body and pedicle or when they involved the vertebra extensively (5). Rib lesions were categorized as malignant when they presented as elongated uptake, categorized as benign (fractures) when vertically they involved several ribs. The additional criteria for the CT component of fused SPECT/CT interpretation were that osteolytic lesions without sclerosis and osteoblastic lesions were considered malignant and that sclerotic lesions with spondylophytes and disk space narrowing or in the subchondral region of a joint together with joint space narrowing, subchondral cysts, and osteophytes were considered benign (6). Clinical and imaging (scintigraphic, CT or MRI) follow-up for at least 6 months (all patients), including tumor markers such as CA 15-3 were used as the reference standard. Increase in the size or changes in the character (osteolytic to sclerotic) during therapy were considered positive for malignancy, whereas lesions with unchanged size and character over 6 months in the absence of treatment were regarded as benign. 


\section{Statistical Analysis}

A lesion-based analysis was performed. Sensitivity, specificity and accuracy for the diagnosis of malignant or benign bone lesions were calculated using standard statistical formulas for planar BS, SPECT and SPECT/CT. Fisher's exact test was used to determine statistical significance for the comparison of planar BS, SPECT and SPECT/CT. A p value <0.05 was considered as statistically significant.

\section{RESULTS}

A total of 72 lesions were evaluated in 23 patients. Four patients had single lesions, four had two lesions, six had three lesions, four had four lesions, four had five lesions and one had six lesions. These were located in the spine-51, ribs-8, sternum-4, clavicle-2, humerus-1, pelvis-5 and femur1. Of the 72 lesions, 59 were metastatic and 13 were benign lesions based on the reference standard. In 57 of the 72 evaluated lesions (79.17\%), the results of planar BS, SPECT and SPECT/CT were concordant; 52 of the 57 lesions were interpreted as malignant and 5 lesions as benign. SPECT/CT, SPECT and planar BS were discordant in 15 of 72 lesions (20.83\%). The consensus reading of SPECT/CT images changed the image interpretation of 15 planar BS scans and 9 SPECT scans: 7 lesions previously interpreted as benign (false-negative on both planar \&SPECT) were re-classified as malignant (Figure 1,2), and 8 lesions previously interpreted as malignant (8 on planar BS, and 2 of 8 on SPECT) were re-evaluated as benign. These 7 malignant lesions in 3 patients could be correlated with osteolytic metastases on CT, and the 8 benign lesions were old fracture of rib, osteoarthritis, spondylosis and spondylarthrosis of the spine. An example is shown in Figure 3. 


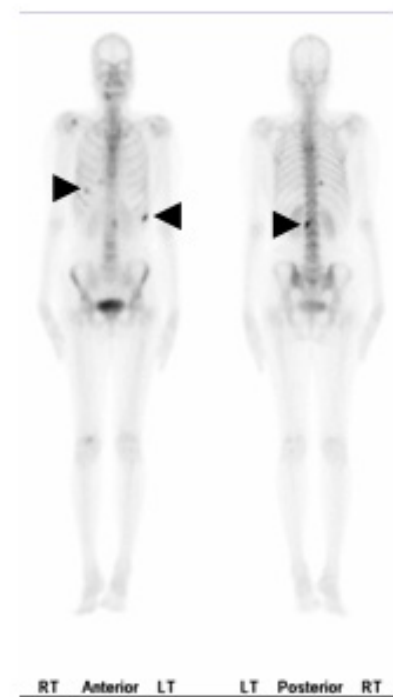

A

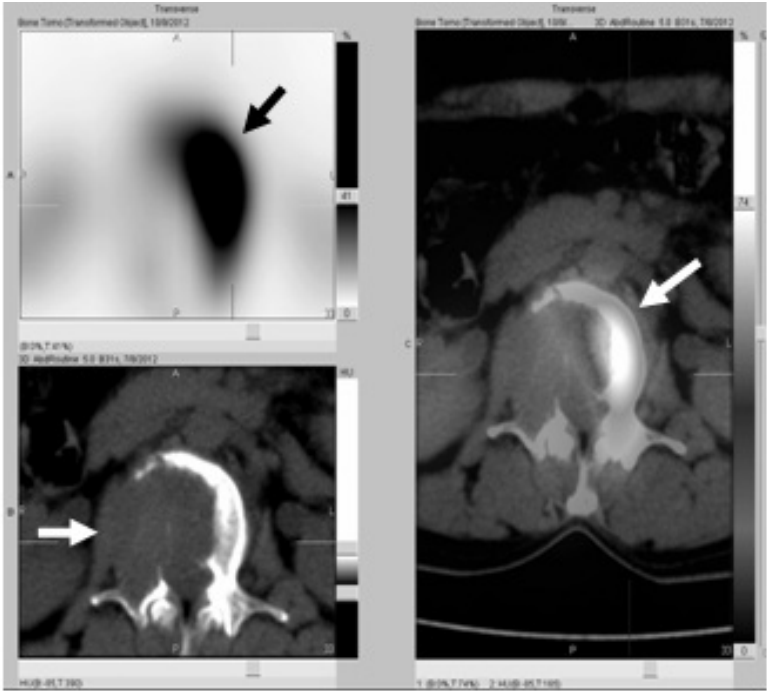

B

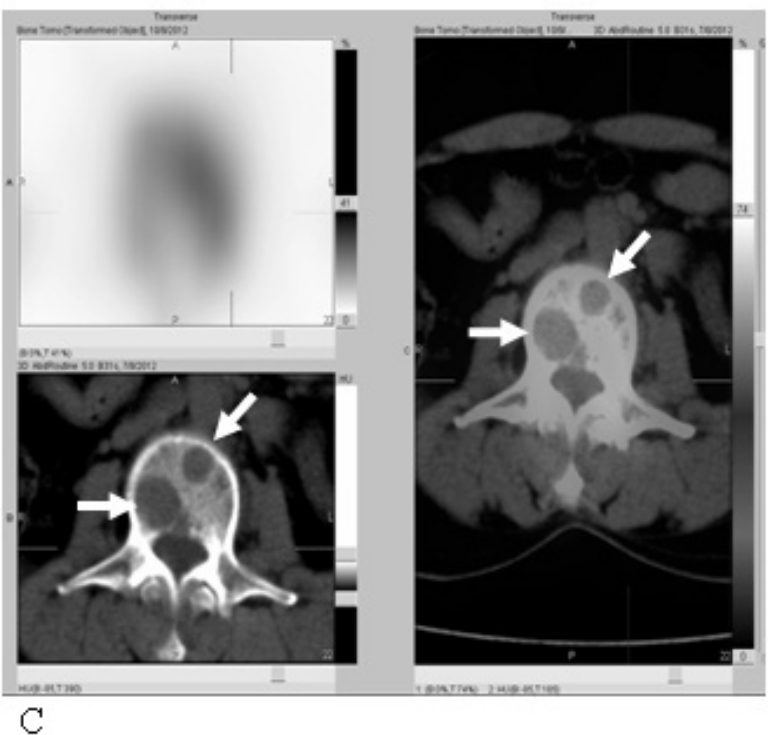

Figure 1. A 56-year-old woman who underwent staging 1 week after resection of right breast cancer and axillary lymph node metastasis. (A) Anterior and posterior images of planar BS showed foci of increased tracer uptake in right $7^{\text {th }}$ and left $9^{\text {th }}$ ribs and left lateral aspects of $1^{\text {st }}$ lumbar vertebra (arrow heads) suggesting metastases. (B) Axial SPECT image (left upper image) showed intense tracer uptake in the left lateral part of the vertebra, where CT (left lower image) showed large osteolytic lesion involving greater part of the vertebral body associated with osteoblastic reaction at the left lateral border, and fused SPECT/CT (right image) showed mixed osteolytic-osteoblastic metastasis in the vertebral body. (C) Axial SPECT (upper image) and planar BS (A) images showed no significant tracer uptake in the $2^{\text {nd }}$ lumbar vertebra of the same patient but the CT and SPECT/CT images showed two additional osteolytic metastases, which were not shown on planar BS and SPECT. Follow up bone scans and CT scans helped confirm the multiple bone metastases. 

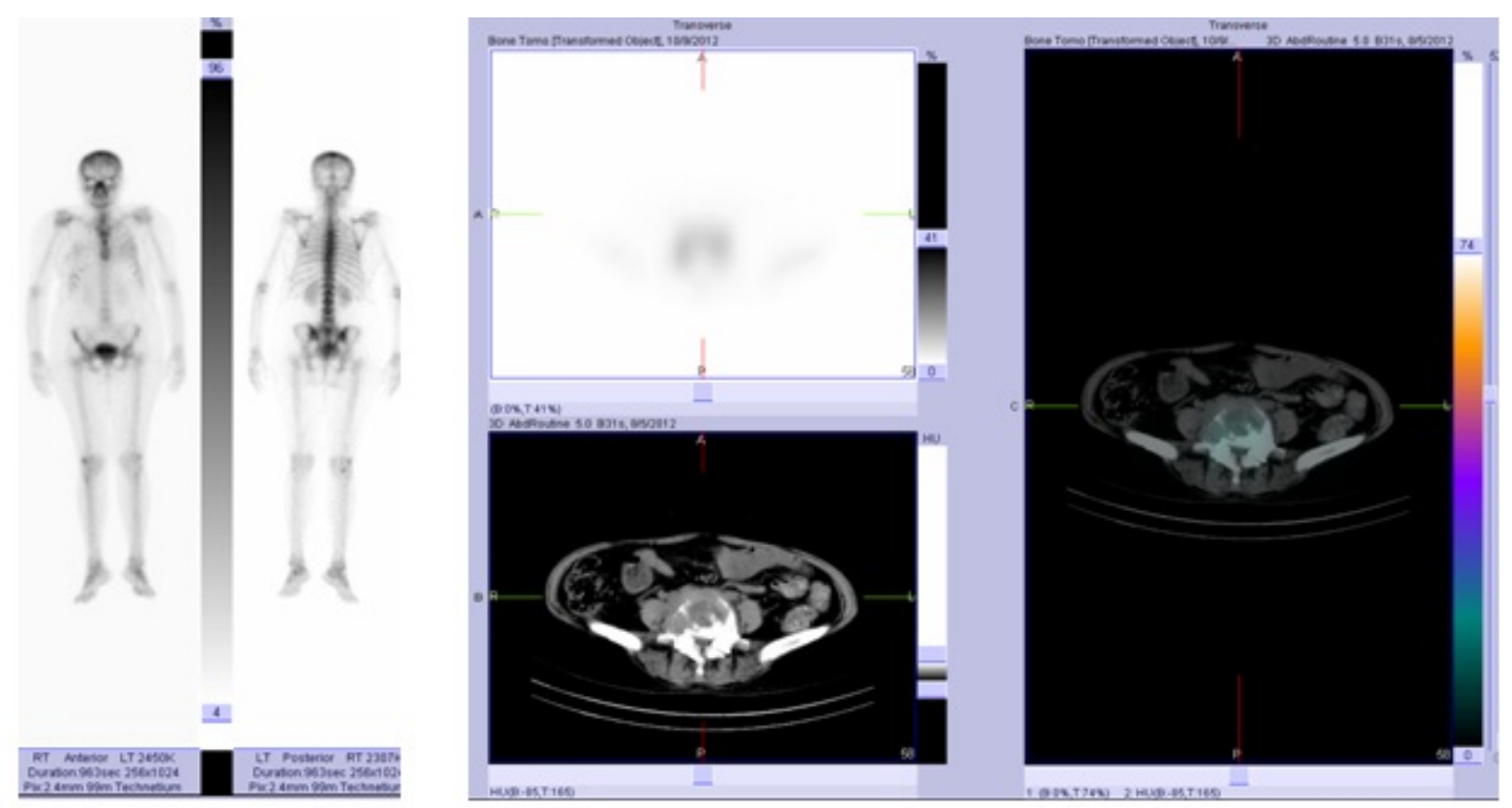

Figure 2. A single metastasis missed on planar BS and SPECT in a 49-year-old patient with breast carcinoma at diagnosis. (A) Anterior and posterior planar BS and (B) axial SPECT (left upper image), which were interpreted as negative for bone metastasis. (B) Axial CT (left lower image) and SPECT/CT identified osteolytic metastasis in L5 vertebra (marked by arrows). Later bone scans and CT scans confirmed the bone metastasis.
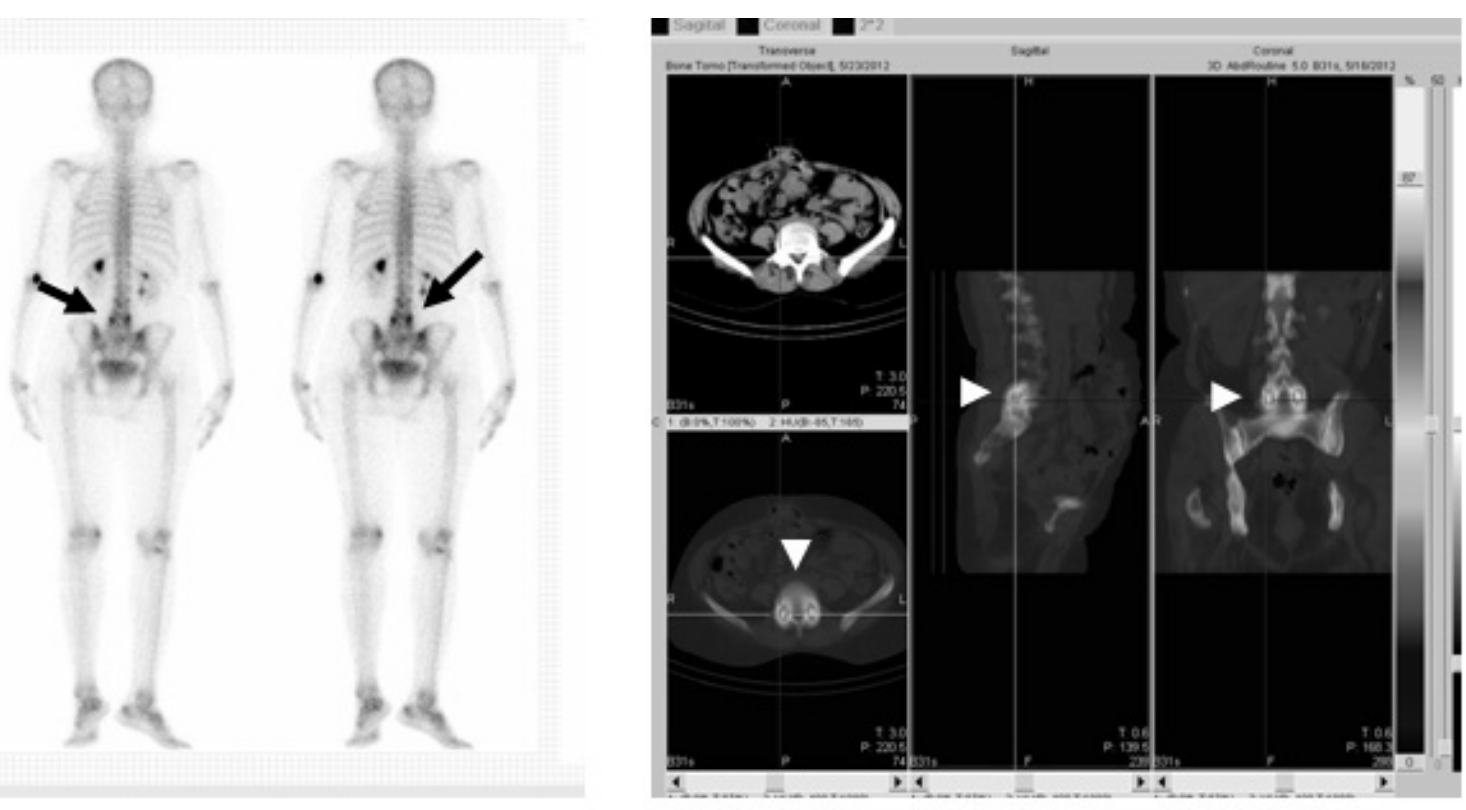

Figure 3. Images of a 63-year-old woman with breast cancer. (A) Anterior and posterior planar BS and (B) axial SPECT (left upper image) showed bilateral foci of increased tracer uptake in L4 vertebra (arrows). (B) Axial CT (left lower image) showed no apparent bone lesion. (B) Axial, saggital and coronal fused images (SPECT/CT) showed precise localization of abnormal tracer uptake in facet joints of both sides (arrows). This finding was suggestive of spondylarthrosis. Imaging and clinical follow-up confirmed the absence of bone metastasis. 
Diagnostic performances of planar BS, SPECT and SPECT/CT are summarized in Table 1. The sensitivity and specificity for differentiation of benign and malignant bone lesions were $88 \%$ and $39 \%$ for planar BS, $88 \%$ and $85 \%$ for SPECT, and $100 \%$ and $100 \%$ for SPECT/CT on a lesionbased analysis. Sensitivity of SPECT/CT was significantly superior to planar BS and SPECT (p $=0.012$ ). Sensitivity of SPECT and planar BS was the same. A specific diagnosis was made with an accuracy of $79 \%$ with planar BS, $83 \%$ with SPECT, and 100\% with SPECT/CT. SPECT/CT was significantly superior to planar BS and SPECT $(\mathrm{p}<0.0001$ and $\mathrm{p}=0.003$, respectively) for specific diagnosis. It was higher (83\% vs $79 \%)$ but not statistically significant when compared between SPECT and planar BS ( $\mathrm{p}=0.263)$. However, specificity of SPECT was significantly higher compared with planar BS $(\mathrm{p}=0.012)$. The results provide evidence of a substantial incremental increase in diagnostic accuracy using SPECT/CT over planar imaging or SPECT alone.

\section{Table 1: Comparison of Planar BS, SPECT and SPECT /CT for detecting metastatic bone lesions, a lesion-based analysis $(n=72)$.}

\begin{tabular}{|c|c|c|c|c|c|c|c|c|c|}
\hline Modaliy & $\mathrm{TP}$ & $\mathrm{TN}$ & $\mathrm{FP}$ & FN & $\begin{array}{c}\text { Sensitity } \\
\%\end{array}$ & $\begin{array}{c}\text { Specificity } \\
\%\end{array}$ & $\begin{array}{c}\text { Accuray } \\
\%\end{array}$ & $\begin{array}{c}\text { PPV } \\
\%\end{array}$ & $\begin{array}{c}\text { NPV } \\
\%\end{array}$ \\
\hline $\begin{array}{l}\text { Planar } \\
\text { BS }\end{array}$ & 52 & 5 & 8 & 7 & 88 & 39 & 79 & 90 & 60 \\
\hline SPECT & 52 & 11 & 2 & 7 & 88 & 85 & 83 & 91 & 90 \\
\hline $\begin{array}{l}\text { SPECT/ } \\
\text { CT }\end{array}$ & 59 & 13 & 0 & 0 & 100 & 100 & 100 & 100 & 100 \\
\hline
\end{tabular}

$\mathrm{BS}=$ bone scintigraphy, $\mathrm{TP}=$ true-positive, $\mathrm{TN}=$ true-negative, $\mathrm{FP}=$ false-positive, $\mathrm{FN}=$ falsenegative.

\section{DISCUSSION}

The development of hybrid SPECT/CT has been popularized by the recent implementation of spiral CT scanners. These techniques can adapt the field of view of the CT scan to the SPECT findings. In the current study, our results demonstrated an improvement in the diagnostic efficacy 
of bone scanning with the addition of SPECT/CT for the evaluation of bony lesions in patients with breast cancer. SPECT/CT was more sensitive and specific than bone scintigraphy, particularly when compared with planar BS but also when compared with SPECT. One key observation was the accurate diagnosis of seven metastatic lesions missed by both planar and SPECT scintigraphy. The addition of SPECT improves the diagnostic accuracy of planar BS. It helps in accurate localization of the tracer activity in bony structures with complex anatomy, such as the spine. The specificity of SPECT in this study was $85 \%$. It was superior to planar BS for the characterization of focal bony lesions $(\mathrm{p}=0.012)$. However, 7 lesions still remained undetected which were correctly diagnosed as malignant by SPECT/CT. With SPECT/CT, 100\% of diagnoses were made with the highest confidence level (planar BS, 79\%; SPECT, 83\%). Several studies have compared the efficacy of SPECT and SPECT/CT in differentiating benign from malignant bone disease (2,7). Using SPECT and a low-dose CT scanner, Horgeret al. correctly classified over $85 \%$ of equivocal foci, compared to $36 \%$ using SPECT alone (5). More recently, a retrospective review of cancer patients by Romer et al. found fused SPECT/CT would be helpful in reaching a definite diagnosis in over $90 \%$ of lesions considered equivocal on SPECT (4). Over $60 \%$ of these lesions were located in the cervical, thoracic or lumbar spine. Utsunomiya et al. compared diagnostic confidence in side-by-side interpretation of CT and SPECT data with confidence in interpretation of fused images of patients with suspected bone metastasis and found that interpretation of fused images increases diagnostic confidence (8). The information gained by SPECT and CT image fusion was greater than the sum of their individual contributions.

The addition of a SPECT/CT scan to the imaging protocol of bone scintigraphy for patients with carcinoma of the breast increased the diagnostic confidence of the reporting physicians in this study. A total of $20.83 \%$ of 72 lesions reported by the physicians on planar and SPECT scans were rated as equivocal or benign, while all of the lesions were accurately classified by SPECT/CT. The combination of SPECT with CT for accurate localization and lesion characterization led to the significant improvements in diagnostic accuracy seen in this study. These trends are consistent with previous research in the cancer population as a whole which has demonstrated reviewers to be significantly more confident in their SPECT/CT diagnoses than those made on planar scintigraphy or $\operatorname{SPECT}(8,9)$. In a study at the Department of nuclear medicine at University Hospital Zurich, a specific diagnosis was made with planar scintigraphy in $64 \%$ of lesions, with SPECT in $86 \%$, and with SPECT/CT in all lesions (9). This observation is 
likely to account for the greater improvement reported in this study highlighting the incremental benefit of SPECT/CT over SPECT alone, in comparison with planar bone scintigraphy.

SPECT/CT was especially beneficial for the five patients where it resulted in a change of diagnosis. If SPECT/CT had not been included in the imaging protocol for patients in this sample, our results indicate that two false positives and seven false negatives would have occurred. Clearly there are implications for treatment and prognosis of localized versus metastatic breast cancer. A diagnosis of metastatic disease has profound consequences for the patient, their quality of life and overall survival. The shifts in diagnosis described above and the findings on follow-up suggest the potential effect of SPECT/CT on sensitivity and specificity. There was complete agreement between SPECT/CT and follow-up imaging for 15 lesions in 8 patients. This included the five patients in whom SPECT/CT resulted in a change in diagnosis. Previous work in other patient cohorts supports this finding $(4,10,11)$. Findings on SPECT/CT led to a specific diagnosis in all patients. Often patients with malignant tumors have unclear pain in the skeleton. In theses situations, it does not suffice to rule out metastasis. The cause of benign focal uptake of radiotracer should be evaluated carefully because many underlying diseases can influence treatment. Fused images facilitated the differentiation of benign from metastatic lesion that were difficult to differentiate on scintigraphic scans. Here it was found that fused images were especially useful for differentiating osteoarthritis and spondylarthrosis of the spine. Metastatic foci that represented slight changes and that were overlooked on two separate sets of scintigraphic and CT images could be detected on fused images.

The present study is not without its limitations. This study and results are limited by the relatively small population and the lack of a histologic gold standard. The ideal gold standard for any investigative analysis is the histological confirmation of the findings. We confirmed bone metastasis on the basis of imaging studies and the subsequent clinical course. This is a limitation of this study, but clinical and imaging follow-up for lesions is currently the acceptable gold standard as histological data for all lesions are impractical in these patients. In certain cases it would have been unethical to investigate all detected lesions by invasive procedures, and radiotherapy or chemo radiotherapy was usually started based on criteria, which are routinely used at our institution. Another limitation is related to the retrospective nature of this study and producing an enrollment bias such that only positive bone scan patients were included for SPECT/CT imaging. Further prospective studies in larger numbers of patients are warranted in order to more precisely define its clinical role and accuracy in the detection of bone metastasis. 
Cost-benefit analysis is also an essential step to understanding the implications of SPECT/CT for the diagnosis and management of carcinoma of the breast.

In conclusion, the results of the present study indicate that the addition of SPECT/CT resulted in a significant reduction of false-negative reports. A definitive diagnosis was given in the majority of the cases due to the improved diagnostic accuracy compared to planar or SPECT scintigraphy alone in breast cancer patients with suspected bone metastases. When available SPECT/CT is indicated in patients in whom correct interpretation of scintigraphic studies is expected to alter the patient's management.

\section{ACKNOWLEDGEMENTS}

The SPECT/CT system Symbia T2 was provided by Siemens Medical Solutions. We appreciate Dr. Md. Aminul Islam, Asstt. Professor of Radiotherapy, $\mathrm{MMCH}$, for referring patients. We thank Nazim Uddin and Abdul Karim for their technical supports in performing the SPECT/CT studies. No potential conflict of interest relevant to this article was reported.

\section{REFERENCES}

1. Hamaoka T, Madewell JE, Podoloff DA, Hortobagyi GN, Ueno NT. Bone imaging in metastatic breast cancer. J ClinOncol. 2004;22:2942-2953.

2. Savelli G, Maffioli L, Maccauro M, De Deckere E, Bombardeieri E. Bone scintigraphy and the added value of SPECT (single-photon emission computed tomography) in detecting skeletal lesions. Q J Nucl Med 2001; 45: 27-37.

3. Minoves M. Bone and joint sports injuries: the role of bone scintigraphy. Nucl Med Commun 2003;24:3-10.

4. Romer W, Nomayr A, Uder M, Bautz W, Kuwert T. SPECT-guided CT for evaluating foci of increased bone metabolism classified as indeterminate on SPECT in cancer patients. $J$ Nucl Med 2006; 47:1102-1106.

5. Horger M, Eschmann SM, Pfannenberg C, Vonthein R, Besenfelder H, Claussen CD, et al. Evaluation of combined transmission and emission tomography for classification of skeletal lesions. AJRAm J Roentgenol 2004;183: 655-661.

6. Even Sapir E, Metser U, Mishani E, Lievshitz G, Lerman H, Leibovitch I. The detection of bone metastases in patients with high-risk prostate cancer: ${ }^{99 \mathrm{~m}} \mathrm{Tc}-\mathrm{MDP}$ planar bone scintigraphy, single-and multi-field-of-view SPECT, ${ }^{18}$ F-fluoride PET and ${ }^{18}$ F-fluoride PET/CT. J Nucl Med. 2006; 47: 287-297. 
7. Helyar V, Mohan HK, Barwick T, Liveiratos L, Gnanasegaran G, Clarke SE, Fogelman I. The added value of multislice SPECT/CT in patients with equivocal bony metastasis from carcinoma of the prostate.EurJ Nucl MedMol Imaging. 2010; 37: 706- 713.

8. Utsunomiya D, Shiraishi S, Imuta M, Tomiguchi S, Kawanaka K, Morishita S, et al. Added value of SPECT/CT fusion in assessing suspected bone metastasis: comparison with scintigraphy alone and nonfusedscintigraphy and CT. Radiology 2006;238:264-271.

9. Strobel K, Burger C, Seifert B, Husarik DB, Soyka JD, Hany TF. Characterization of focal bone lesions in the axial skeleton: performance of planar bone scintigraphy compared with SPECT and SPECT fused with CT. AJR Am J Roentgenol 2007;188: W467-W474.

10. HorgerM, Bares R. The role of single-photon emission computed tomography/computed tomography in benign and malignant bone disease. SeminNucl Med 2006; 36:286-294.

11. Ndlovu X, George R, Ellmann A, Warwick J. Should SPECT/CT replace SPECT for the evaluation of equivocal bone scan lesions in patients with underlying malignancies? Nucl Med Commun. 2010; 31: 659-665 